\title{
Fermi surface of the skutterudite $\mathrm{CoSb}_{3}$ : Quantum oscillations and band-structure calculations
}

\author{
M. Naumann $\odot,{ }^{1,2, *}$ P. Mokhtari $\odot,{ }^{1,2, *}$ Z. Medvecka, ${ }^{1}$ F. Arnold, ${ }^{1}$ M. Pillaca,,${ }^{3, \dagger}$ S. Flipo, ${ }^{1}$ D. Sun $\odot,{ }^{1}$ H. Rosner, ${ }^{1}$ \\ A. Leithe-Jasper, ${ }^{1}$ P. Gille, ${ }^{3}$ M. Baenitz, ${ }^{1}$ and E. Hassinger ${ }^{1,2,+}$ \\ ${ }^{1}$ Max Planck Institute for Chemical Physics of Solids, 01187 Dresden, Germany \\ ${ }^{2}$ Physik-Department, Technische Universität München, 85748 Garching, Germany \\ ${ }^{3}$ Ludwig-Maximilians-Universität München, 80539 Munich, Germany
}

(Received 4 December 2020; accepted 5 February 2021; published 22 February 2021)

\begin{abstract}
The unfilled skutterudite $\mathrm{CoSb}_{3}$ is a small-gap semiconductor which was predicted to be close to a straininduced transition to a topological-insulator phase passing through a topological quantum critical point. As a starting point to strain measurements, the aim of this work is to characterize the electronic structure of as-grown samples. Two types of samples, one grown in Sb flux and the other by an inclined rotary Bridgman technique, are compared based on structural properties, resistivity, Hall effect, and magnetization. All samples appear metallic with small growth-dependent hole doping. Measurements of quantum oscillations in magnetization and angledependent electronic transport confirm the calculated band structure at ambient pressure. The Fermi surface consists of a single spherical sheet at the $\Gamma$ point, and the purest samples grown by the Bridgman technique have a Fermi energy lying $25 \mathrm{meV}$ below the valence-band edge. Band-structure calculations provide an accurate description of the experimental results. Hence, this compound is suitable for an investigation of topological states under strain.
\end{abstract}

DOI: 10.1103/PhysRevB.103.085133

\section{INTRODUCTION}

Filled skutterudites are interesting solids and exhibit a large number of fascinating properties such as superconductivity, heavy fermion behavior, and Kondo insulator physics [1]. Some of them also have a great potential for thermoelectric applications [2]. In contrast to the filled skutterudites, the unfilled pure skutterudites (such as $\mathrm{CoSb}_{3}$ ) have been less studied. Furthermore, their mostly semiconducting behavior has to be reinterpreted in the light of new findings on topological Dirac and Weyl semimetals. Specifically, there is the prediction that $\mathrm{CoSb}_{3}$ is a so-called proximate Dirac semiconductor which could be tuned by strain towards a topological critical point $[3,4]$. This means that a band touching point (node) in the gap evolves upon the application of strain. The closing and following inversion of the gap results in a Dirac cone with characteristic linear dispersion near the Fermi energy and hence enables the study of a continuous transition through a topological quantum critical point from a normal semiconductor into a topologically nontrivial material $[4,5]$.

\footnotetext{
*These authors contributed equally to this work.

${ }^{\dagger}$ Present address: Faculty of Chemistry and Food Chemistry, Technische Universitt Dresden, 01062 Dresden, Germany.

${ }^{\ddagger}$ Corresponding author: elena.hassinger@cpfs.mpg.de

Published by the American Physical Society under the terms of the Creative Commons Attribution 4.0 International license. Further distribution of this work must maintain attribution to the author(s) and the published article's title, journal citation, and DOI. Open access publication funded by the Max Planck Society.
}

A weak doping (in contrast to the fully filled systems) may have the same effect. For example, even a very small doping with Ni leads to enormous changes in the Seebeck coefficient and mobility [6]. This could be a first indication of a Diraclike dispersion. To understand the tuning effect (via doping or strain), undoped $\mathrm{CoSb}_{3}$ single crystals have to be studied first. Hence, the aim of this study is to confirm the calculated band structure experimentally via quantum oscillations on $\mathrm{CoSb}_{3}$ crystals grown by two different methods, in order to corroborate $\mathrm{CoSb}_{3}$ as a good starting material for investigations of strain-induced topological transitions.

The pristine material fabricated by vapor transport and flux techniques grows slightly hole doped with hole concentrations between $1 \times 10^{16}$ and $1 \times 10^{18} \mathrm{~cm}^{-3}[7,8]$. However, the results of the temperature dependence of the resistivity varied between different studies; some reported metallic behavior, whereas others reported a freeze-out of charge carriers at low temperature [9-12].

The latest investigation into pristine samples was done by analyzing the Shubnikov-de Haas ( $\mathrm{SdH}$ ) effect, which could be observed in this material in high magnetic fields $[7,13]$. These measurements on unoriented crystals did not disagree with $a b$ initio calculations since the results were in accordance with the picture of a single spherical Fermi surface at the Brillouin-zone center [14]. They also observed a spin splitting of the quantum oscillations (QOs), and deduced an appreciably elevated $g$ factor of -10.1 . Unfortunately, their data only allowed a limited analysis due to elevated noise levels in pulsed magnetic fields and the observation of only a few oscillations. In general, a detailed investigation of the electronic structure and properties and their dependence on the growth conditions is still lacking. 
In this study, two sets of as-grown samples are investigated: one grown by a self-flux and a second using an inclined rotary Bridgman (IRB) method [15]. By means of resistivity, Hall effect, magnetization, and QOs therein, we show that the two methods yielded crystals with a reproducible low charge density with dominantly hole charge carriers. The QOs confirm the calculated band structure. We found that samples grown by the IRB method are homogeneous, whereas signs of a sampledependent doping level and $\mathrm{Sb}$ inclusions occurred in the flux-grown samples. We also investigated indium substitution as a means of shifting the Fermi energy to the electron-doped region.

\section{EXPERIMENTAL AND COMPUTATIONAL METHODS}

Sample growth. Two sets of $\mathrm{CoSb}_{3}$ samples were investigated in this study. The first set was prepared by an inclined rotary Bridgman (IRB) method. Details about the IRB technique can be found in Ref. [15]. In this method, an ampule containing cobalt and an excess of antimony relative to the stoichiometric ratio are placed in an oven, with the whole assembly inclined by about $15^{\circ}$ with respect to the horizon. The ampule is rotated at $100 \mathrm{rpm}$, while it is retracted from the oven at a rate of $2.4 \mathrm{~mm}$ per day. Crystals grown by this technique appear as extremely homogeneous with sizes of a few centimeters. These samples are labeled "IRB" in the figures.

The second set of samples was grown by the self-flux method, utilizing an antimony-rich self-flux resulting in crystals of up to $1 \mathrm{~cm}$ size, and showing facets along certain high-symmetry planes of the cubic lattice. However, the bulk of the material hosted some voids of millimeter size and $\mathrm{Sb}$ inclusions, as observed via quantum oscillations as described below. Powders of the elements Co (99.998 wt.\%, Chempur) and $\mathrm{Sb}\left(99.999 \%\right.$, Chempur) were mixed in the $\mathrm{CoSb}_{3}$ stoichiometric ratio, compacted in steel dies without the use of lubricants, and placed in glassy carbon crucibles, which were then sealed into quartz ampoules under a pressure of $200 \mathrm{mbar}$ of Ar gas. They were reaction sintered at $600{ }^{\circ} \mathrm{C}$ for 5 days. In a second step, several grams of these precursors were ground and mixed with an excess of $\mathrm{Sb}$ in molar ratios $\mathrm{CoSb}_{3}: \mathrm{Sb}$ (1:913), compacted, placed in glassy carbon crucibles, and sealed into quartz ampoules. Crystal growth was accomplished by heating up to $700{ }^{\circ} \mathrm{C}$ within 6 hours, followed by a soaking time of 24 hours, then heating to $900{ }^{\circ} \mathrm{C}$ with soaking for 24 hours, followed by cooling to $650{ }^{\circ} \mathrm{C}$ within 10 days. After crystal growth, the excess antimony was removed by sublimation at $550{ }^{\circ} \mathrm{C}$ under dynamic vacuum of about $10^{-4}$ mbar. These samples are labeled "flux" in the figures.

With respect to the thermodynamic conditions, the two methods do not differ. Since $\mathrm{CoSb}_{3}$ has a peritectic decomposition temperature of $874{ }^{\circ} \mathrm{C}$, the only way to crystallize it in the binary $\mathrm{Co}-\mathrm{Sb}$ system is from a $\mathrm{Sb}$-rich solution having at least 93 at.\% Sb [15]. Whereas, in the self-flux method, only very weak buoyancy-driven convection assists the slow material transport by diffusion; the main idea of the IRB method is a strong mixing of the solution by forced convection. This may avoid constitutional supercooling, which is the origin of fluid inclusion formation.
In order to study the relation between the lattice properties and the electronic structure of the crystals, all of the batches were investigated by powder $\mathrm{x}$-ray diffraction (PXRD). PXRD measurement of IRB samples was carried out in the diffractometer (X-ray Diffraction System XRD 3003 TT) with Bragg-Brentano geometry, using $\mathrm{Cu}-\mathrm{K}_{\alpha 1}$ radiation $(\lambda=1.5406 \AA)$ at $40 \mathrm{kV}$ and $30 \mathrm{~mA}$. Room-temperature lattice parameters and the $y$ and $z$ parameters giving the antimony position were determined via the Rietveld refinement method. A silicon standard (NIST 640c) was used as an internal standard for the determination of the lattice parameters. PXRD measurements on powdered samples of the self-fluxgrown crystals were done with a Guinier Huber G670 image plate camera applying $\mathrm{Co}-\mathrm{K}_{\alpha}$ radiation $(\lambda=1.78896 \AA$ ). The lattice parameters and structural parameters were refined using the WINCSD program package [16]. Chemical analysis of $\mathrm{Fe}$ and of $\mathrm{Ni}$ trace impurities was performed by dissolving crystals in $\mathrm{HCl} / \mathrm{HNO} 3$ (aqua regia) followed by ICP-MS analysis on a Varian ICP-MS 820 system.

For the study with indium doping, we prepared two batches of the nominal composition $\mathrm{In}_{0.05} \mathrm{Co}_{4} \mathrm{Sb}_{12}$ and $\mathrm{In}_{0.1} \mathrm{Co}_{4} \mathrm{Sb}_{12}$ by the IRB technique, which are labeled in this text as batches "In0.05-CoSb 3 " and "In0.10-CoSb 3 ," respectively. By using the electron probe micro analysis (EPMA) technique, it was not possible to determine the real indium content of those samples.

DFT. Relativistic density functional theory (DFT) electronic-structure calculations were performed using the full-potential FPLO code [17,18], version fplo18.00-52. For the exchange-correlation potential, within the generalized gradient approximation (GGA), the Perdew-Burke-Ernzerhof parametrization [19] was chosen. These results were compared to the local density approximation (LDA) [20]. The spin-orbit (SO) coupling was treated nonperturbatively, solving the four-component Kohn-Sham-Dirac equation [21]. Calculations were done using experimental room-temperature lattice parameters, as indicated in the text and figure captions. The calculations were carried out on a well-converged mesh of 27000 points $(30 \times 30 \times 30$ mesh, 1256 points in the irreducible wedge of the Brillouin zone).

Magnetization and resistivity. Magnetization as well as resistivity measurements were used to study the electronic structure via quantum oscillations. Magnetization measurements were performed in a Quantum Design (QD) superconducting quantum interference device (SQUID) vibrating-sample magnetometer (VSM) at temperatures down to $2 \mathrm{~K}$ and magnetic fields up to $7 \mathrm{~T}$.

Resistance measurements were mostly performed in the QD physical properties measurement systems (PPMS) at fields up to $9 \mathrm{~T}$ or $14 \mathrm{~T}$. Six contacts on the sample allowed for a simultaneous measurement of the resistivity and the Hall resistivity. A typical current of $5 \mathrm{~mA}$ was applied along the [100] direction of the crystal. The magnetic field was applied along the $b$ axis, perpendicular to the current direction. The Hall resistivity $\rho_{y x}$ is related with the magnetic field $B$ and the carrier density $n_{\text {Hall }}$ via $\rho_{y x}=\left(n_{\text {Hall }} e\right)^{-1} B$, where $e$ is the electron charge. It is positive for holelike charge carriers and negative for electronlike carriers. A rotator probe was also utilized for a full rotation study of the quantum oscillations. For a few investigations of the high-field and low-temperature 
TABLE I. Impurity concentrations of $\mathrm{Fe}$ and $\mathrm{Ni}$, and lattice constant $a$ including the $\mathrm{Sb}$ position by $y$ and $z$ values, as determined by chemical analysis and PXRD measurements, respectively. Numbers 1 to 5 are samples grown by the flux technique, and IRB is from the IRB technique, where all measured samples showed the same values.

\begin{tabular}{|c|c|c|c|c|c|}
\hline Sample & $\mathrm{Fe}(\mathrm{ppm})$ & $\mathrm{Ni}(\mathrm{ppm})$ & $a(\AA)$ & $y$ & $z$ \\
\hline Flux No. 1 & $26(3)$ & $31(5)$ & $9.0363(5)$ & $0.3329(3)$ & $0.1568(3)$ \\
\hline Flux No. 2 & $13(1)$ & $12(2)$ & $9.0398(3)$ & $0.3347(3)$ & $0.1563(3)$ \\
\hline Flux No. 3 & $<13$ & $<3$ & $9.0376(5)$ & $0.3331(3)$ & $0.1567(3)$ \\
\hline Flux No. 4 & $46(5)$ & $127(7)$ & $9.0362(4)$ & $0.3336(2)$ & $0.1563(2)$ \\
\hline Flux No. 5 & & & $9.0434(5)$ & $0.3325(3)$ & $0.1560(3)$ \\
\hline IRB & & & $9.0362(1)$ & $0.3343(9)$ & $0.1574(1)$ \\
\hline $\mathrm{In} 0.05-\mathrm{CoSb}_{3}$ & & & $9.0373(1)$ & $0.3340(9)$ & $0.1572(1)$ \\
\hline $\mathrm{In} 0.10-\mathrm{CoSb}_{3}$ & & & $9.0381(1)$ & $0.3344(8)$ & $0.1570(9)$ \\
\hline
\end{tabular}

properties, two ${ }^{3} \mathrm{He}-{ }^{4} \mathrm{He}$ dilution refrigerators were operated, where Stanford Research SR830 and SR850 lock-In amplifiers were mostly used. One of the dilution fridges was equipped with a low-temperature transformer (LTT). In this system, a National Instruments PXI controller for signal generation and $\mathrm{AD}$ conversion was employed, together with a subsequent software lock-in procedure.

Quantum-oscillation analysis. Since the cross-sectional area of the Fermi surface perpendicular to the applied magnetic field $A_{\perp}$ is proportional to the frequency $F$ of the quantum oscillations as $A_{\perp}=(2 \pi e / \hbar c) F$, the change of the oscillation's frequency with angle will reveal the Fermisurface (FS) structure. Here, $e$ is the electron charge, $\hbar$ is Planck's constant divided by $2 \pi$, and $c$ is the light velocity. The de Haas-van Alphen (dHvA) oscillations in the magnetization and Shubnikov-de Haas oscillations in the resistivity were analyzed by removing a field-dependent background using a polynomial function. The fast Fourier transform (FFT) was applied to the oscillatory signal as a function of inverse field using a Kaiser window with $a=6$. For a given spherical Fermi surface, the QO frequency $F$ is related to the $k_{F}$ by $k_{F}=\sqrt{F / F_{0} \pi}$, and $F_{0}=10476 \mathrm{~T}^{2}$.

The temperature dependence of the peak amplitude in the FFT spectrum was used to determine the cyclotron effective mass $m^{*}$ of the charge carriers. Since the field window is rather wide with respect to the field dependence of the amplitude, special care had to be taken not to underestimate the effective mass. The strategy to avoid this is detailed in Ref. [22], where the field dependence by the Dingle term in the Lifshitz-Kosevich term is taken into account. The thermal damping factor $A(T)$ is the Lifshitz-Kosevich temperature dependence, which has the form of $A(T)=\xi / \sinh \xi$ with $\xi=\pi^{2} m^{*} k_{B} T / \mu_{B} B$. Here, $k_{B}$ is the Boltzmann constant and $\mu_{B}$ is the Bohr magneton. $D(1 / B)$ is the Dingle term determined as $D(1 / B)=\exp \left(-14.69 T_{D} m^{*} 1 / B\right)$ using the Dingle temperature $T_{D} . T_{D}$ is extracted from the width of the FFT line shape [23].

Moreover, the mean free path $\lambda$ was obtained from $T_{D}$, which is related to the scattering time $\tau$ by $T_{D}=\hbar /\left(2 \pi k_{B} \tau\right)$. Substituting the scattering time with the mean free path $\lambda$, the Fermi velocity $v_{F}$, and the latter with $v_{F}=\hbar k_{F} / m^{*}$ yields $\lambda=\hbar^{2} k_{F} /\left(2 \pi m^{*} k_{B} T_{D}\right)$.

\section{RESULTS AND DISCUSSION}

Lattice parameters. The lattice parameters and antimony positions are presented in Table I. See, also, the crystal structure in Fig. 1(a). The largest deviations between the batches appear in the antimony position $z$ with almost $1 \%$ difference. The IRB-grown samples show a lattice parameter in agreement with the smallest parameter from the flux growth. (a)

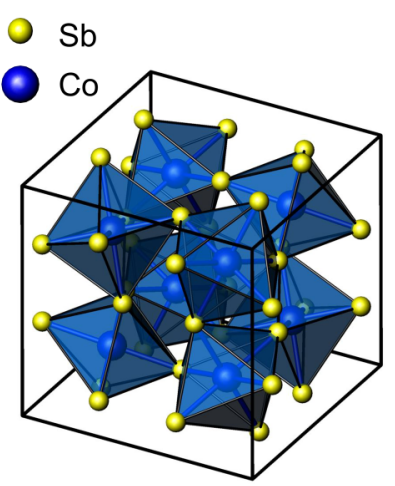

(b)

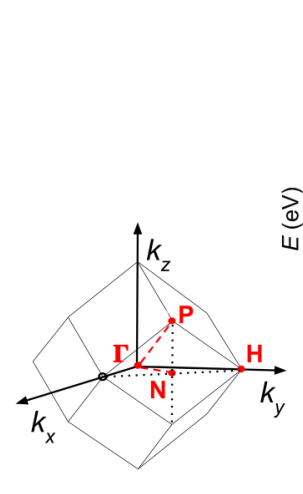

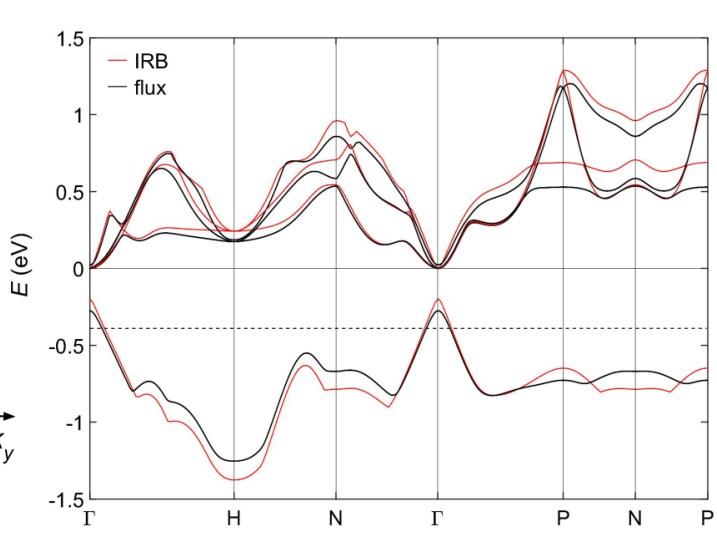

(c)

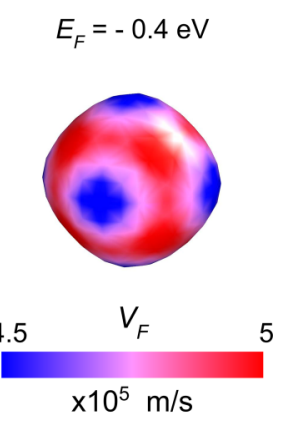

FIG. 1. (a) Crystal structure of $\mathrm{CoSb}_{3}$. (b) Band structure along the high-symmetry directions using structural parameters from Table I for the IRB sample and batch flux No. 5. The indexing is shown in the Brillouin zone. The Fermi energy of the Fermi surface shown in (c) is indicated by a dashed line. (c) The Fermi surface for the Fermi energy $E_{\mathrm{F}}=-0.4 \mathrm{eV}$. The color code illustrates the magnitude of the Fermi velocity on the surface. 

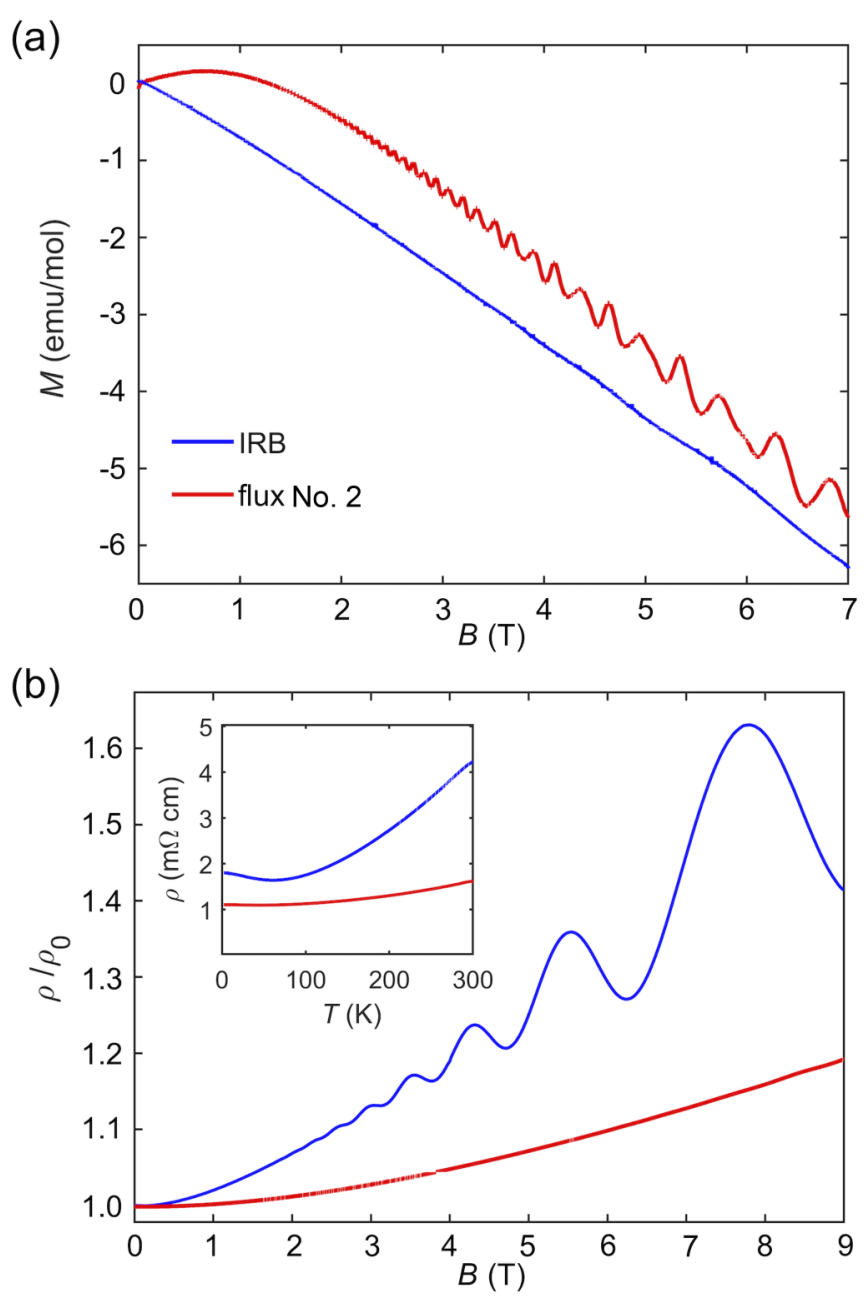

FIG. 2. (a) Magnetization $M$ vs magnetic field $B$ at $T=2 \mathrm{~K}$ for a flux-grown sample from the batch flux No. 2 (red curve) and one sample grown by the IRB technique (blue curve). Note that some flux-grown samples show a paramagnetic behavior on top of the dominantly diamagnetic signal, such as the sample shown here. (b) Normalized resistivity vs magnetic field at $2 \mathrm{~K}$ for the same samples. $\rho_{0}=\rho(B=0 T)$ for IRB and flux samples are equal to $1.798 \mathrm{~m} \Omega \mathrm{cm}$ and $1.041 \mathrm{~m} \Omega \mathrm{cm}$, respectively. The inset shows the temperature dependence of the zero-field resistivity of these samples, which confirms the metallic behavior. A slight increase in the resistivity at the lowest temperatures was observed for the IRB samples.

DFT. First, we calculated the band structure using different levels of approximation. A comparison of GGA and LDA as well as the effect of the spin-orbit coupling (SOC) can be seen in the Supplemental Material [25]. These different approximations give essentially the same results: Three conduction bands (almost) meet at the band edge at the $\Gamma$ point. They are separated from a single valence band by a direct gap of around $250 \mathrm{meV}$. The effect of a difference in lattice parameters on the band structure is depicted in Fig. 1(b), using the experimental room-temperature lattice parameters from Table I of the IRB-grown samples (red) compared with the batch flux No. 5 (black) which had the largest difference of the lattice parameter and antimony position. Near the gap, the band structures are very similar, with a slightly enhanced gap for larger lattice parameter and smaller Sb position.
Overall, the DFT results are robust. Neither different DFT functionals, the in- or exclusion of spin-orbit coupling, nor reasonable changes in the structural parameters have a significant influence on the results when the Fermi energy is near the gap.

If the Fermi energy is below the gap (hole doping), the DFT calculations predict only one single band that gives rise to a single, spherical Fermi surface, as depicted in Fig. 1(c), leading to one quantum-oscillation frequency. If the Fermi energy is in the electron-doped region, however, three Fermi-surface sheets should appear with a shape differing from a simple sphere (see the paragraph about In-doped samples).

\section{A. Stoichiometric samples}

Magnetization. The magnetization of $\mathrm{CoSb}_{3}$ can be found in Fig. 2(a) for two samples: a sample from the batch flux No. 2 and an IRB-grown sample. The samples are diamagnetic with a susceptibility of around $\chi_{m} \approx-8.6 \times$ $10^{-5} \mathrm{emu} / \mathrm{mol}$ Oe.

Many of the flux-grown samples show signs of paramagnetism on top of a clear diamagnetic background. This can be explained by magnetic impurities, i.e., the magnitude of the paramagnetic contribution fits well with the order of magnitude of a few ppm $\mathrm{Fe}$ and $\mathrm{Ni}$, which were found via chemical analysis (see Table I). For example, in the case of the flux-grown sample No. 2, the chemical analysis gives $13 \mathrm{ppm}$ $\mathrm{Fe}$ and $12 \mathrm{ppm} \mathrm{Ni}$. In comparison, a fit of the magnetization with the sum of a Brillouin function and a diamagnetic background returns a magnetic impurity concentration of $50 \mathrm{ppm}$ and $74 \mathrm{ppm}$ for the total angular momentum quantum number $J$ equal to 2 and $5 / 2$, for $\mathrm{Fe}$ and $\mathrm{Ni}$, respectively. On top of the para- and diamagnetic background, dHvA oscillations can be seen. In our samples, we find that those oscillations are more pronounced in most of the flux-grown samples compared to the IRB-grown ones, as depicted in Fig. 2(a). It is worth noting that despite the expected strong sensitivity of quantum oscillations to impurity scattering, no systematic variation of the QO amplitude (and extracted mean free path) with the magnetic impurity concentration was found (see Tables I and II). The origin of this lies in the fact that the QOs observed in most of the flux-grown batches stem from inclusions of pure $\mathrm{Sb}$ from the flux, as can be inferred from the analysis of the effective mass that will be described below. Sb is a semimetal and therefore exhibits very strong de Haas-van Alphen oscillations that can dominate the oscillatory magnetization signal even when the volume of the $\mathrm{Sb}$ inclusions is small. Hence, the given mean free path in Table II is the one in those Sb inclusions and independent of the concentration of $\mathrm{Fe}$ and $\mathrm{Ni}$ impurities most likely incorporated directly in the $\mathrm{CoSb}_{3}$.

The IRB samples are free from $\mathrm{Sb}$ inclusions and magnetic impurities. Visible quantum oscillations are intrinsic to weakly impurity-doped $\mathrm{CoSb}_{3}$.

Resistivity. The resistivity as a function of temperature can be seen in the inset of Fig. 2(b). It drops upon cooling the sample down from room temperature, reflecting a metallic behavior. This means that the natural doping during crystal growth in $\mathrm{CoSb}_{3}$ is large enough to tune the Fermi energy away from the gap. Unlike some reports [8-11], all 
TABLE II. Overview of our samples and their electronic properties: QO frequency $F$, mean free path $\lambda$, effective mass $m^{*}$ in units of the free electron mass $m_{e}$, carrier density $n_{Q O}$ obtained from quantum oscillations, and carrier density from the Hall measurements $n_{\text {Hall }}$.

\begin{tabular}{|c|c|c|c|c|c|c|}
\hline Sample & Observed effect & $\begin{array}{c}F(B \|[100]) \\
(\mathrm{T})\end{array}$ & $\begin{array}{c}n_{Q O} \\
\left(10^{18} \mathrm{~cm}^{-3}\right)\end{array}$ & $\begin{array}{c}n_{\text {Hall }} \\
\left(10^{18} \mathrm{~cm}^{-3}\right)\end{array}$ & $\begin{array}{c}m^{*} / m_{e} \\
\left(10^{-3}\right)\end{array}$ & $\begin{array}{c}\lambda \\
(\mathrm{nm})\end{array}$ \\
\hline \multicolumn{7}{|c|}{ QOs from $\mathrm{CoSb}_{3}$} \\
\hline IRB S1 & $\mathrm{dHvA}$ and $\mathrm{SdH}$ & 19.0 & 0.47 & 0.55 & 105 & 35 \\
\hline IRB S2 & $\mathrm{dHvA}$ and $\mathrm{SdH}$ & 20.4 & 0.52 & 0.65 & 93 & 30 \\
\hline IRB S3 & $\mathrm{dHvA}$ and $\mathrm{SdH}$ & 19.2 & 0.48 & & 95 & 30 \\
\hline Flux No. $4 \mathrm{~S} 1$ & $\mathrm{SdH}$ & 57 & 2.6 & 10 & 122 & 30 \\
\hline Flux No. $4 \mathrm{~S} 2$ & $\mathrm{SdH}$ & 79 & 4.0 & 30 & 140 & 30 \\
\hline \multicolumn{7}{|c|}{ QOs from Sb inclusions } \\
\hline \multirow[t]{2}{*}{ Flux No. $1 \mathrm{~S} 1$} & $\mathrm{dHvA}$ & 63 & 2.8 & 26 & 63 & 75 \\
\hline & & 86 & 4.5 & & & \\
\hline \multirow[t]{3}{*}{ Flux No. $1 \mathrm{~S} 2$} & $\mathrm{dHvA}$ & 63 & 2.6 & & 69 & 81 \\
\hline & & 78 & 3.9 & & 83 & 120 \\
\hline & & 108 & 6.3 & & 110 & 120 \\
\hline \multirow[t]{2}{*}{ Flux No. 2} & $\mathrm{dHvA}$ and $\mathrm{SdH}$ & 70 & 3.3 & 3.5 & 75 & 220 \\
\hline & & 104 & 6 & & 100 & 140 \\
\hline \multirow[t]{2}{*}{ Flux No. 3} & $\mathrm{dHvA}$ & 66 & 3 & 39 & 70 & 75 \\
\hline & & 85 & 4.4 & & 100 & 120 \\
\hline $\mathrm{In} 0.05-\mathrm{CoSb}_{3}$ & & & & 0.6 & & \\
\hline $\mathrm{In} 0.10-\mathrm{CoSb}_{3}$ & $\mathrm{dHvA}$ and $\mathrm{SdH}$ & 25,40 & & 1.8 & 200 & 20 \\
\hline
\end{tabular}

our as-grown samples are metallic despite having similar lowtemperature resistivity values and charge carrier densities as the published ones. Many samples show a slight increase in the resistivity at the lowest temperatures, with a minimum at around $60 \mathrm{~K}$, such as the IRB sample in the figure. Since there is no complementing negative magnetoresistance, indicative of weak-localization physics [26], this is attributed to the temperature-dependent ionized impurity scattering [27]. The residual resistivity is around $0.2 \mathrm{~m} \Omega \mathrm{cm}$ to $2 \mathrm{~m} \Omega \mathrm{cm}$, and the $R R R=\rho(300 K) / \rho(4 K)$ around 1 to 5 . All the samples show a typical magnetoresistive signal which behaves quadratic at low fields [see Fig. 2(b)]. In contrast to the quantum oscillations in the magnetization, the $\mathrm{SdH}$ oscillations in the resistivity are appreciably more pronounced in the IRB-grown samples. The resistivity signal is dominated by the bulk of the sample consisting of $\mathrm{CoSb}_{3}$ for both types of samples and would not show a strong contribution from $\mathrm{Sb}$ inclusions. Therefore, in the IRB samples (without Sb inclusions), quantum oscillations from $\mathrm{CoSb}_{3}$ consistently appeared in both magnetization and resistivity, whereas samples with $\mathrm{Sb}$ inclusions showed QOs from $\mathrm{Sb}$ in magnetization and sometimes very small QOs in the resistivity. Because these oscillations are so small in the resistivity, we could not analyze the effective masses to unambiguously confirm the $\mathrm{CoSb}_{3}$ origin.

Hall effect. The Hall effect reveals that all as-grown samples without indium are hole doped (Fig. 3). The carrier concentration was determined from the slope of $\rho_{y x}$ and is given in Table II. The Hall signal was usually perfectly linear and only a few samples showed a slight curvature at fields exceeding $5 \mathrm{~T}$. As mentioned above, in the hole-doped region, the Fermi surface is predicted to consist of a single, nearly spherical Fermi surface centered around the $\Gamma$ point. Samples grown by the IRB method yield a hole concentration of $5.5 \times$ $10^{17}-6.5 \times 10^{17} \mathrm{~cm}^{-3}$ corresponding to a QO frequency of $19 \mathrm{~T}$, in perfect agreement with the observed frequency (see below). Crystals grown by the flux method yield larger charge carrier concentrations, $3.5 \times 10^{18}-3.9 \times 10^{19} \mathrm{~cm}^{-3}$.

Quantum oscillations. Quantum oscillations on the complete set of magnetization and resistivity data on various batches and samples were analyzed using the standard proce-

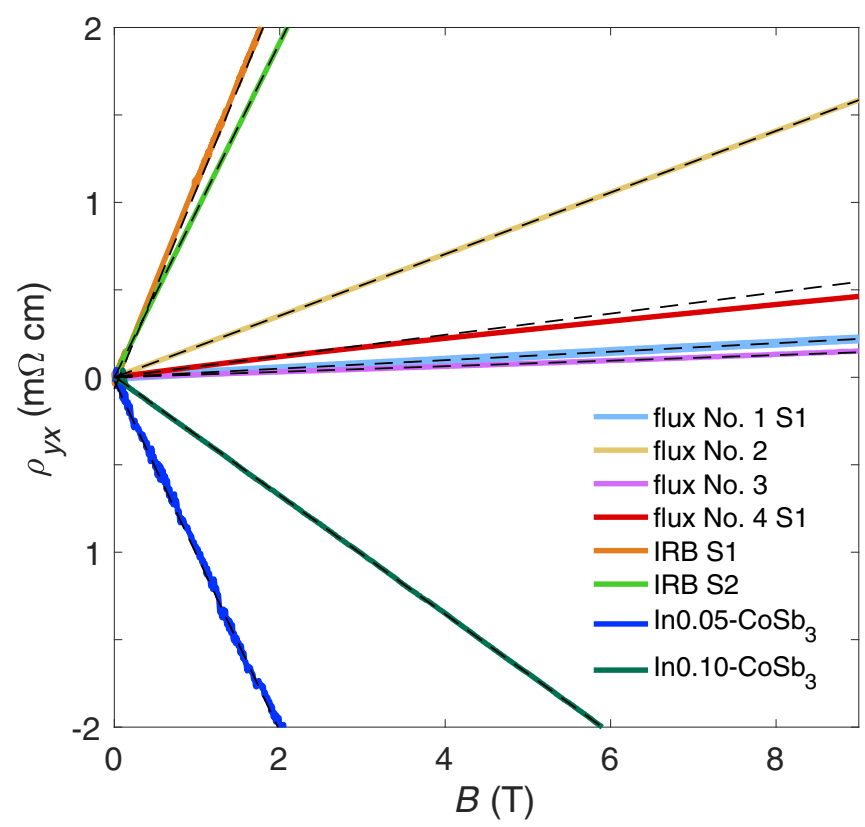

FIG. 3. Hall resistivity $\rho_{y x}$ vs magnetic field $B$ at $T=2 \mathrm{~K}$ for samples from all growth batches (colored data, as indicated). The slope (dashed straight lines) is inversely proportional to the carrier concentration $n_{\text {Hall }}$ given in Table II. As-grown samples have a positive Hall resistivity and are therefore hole doped, whereas In-doped samples appear electron doped. 
(a)

(b)

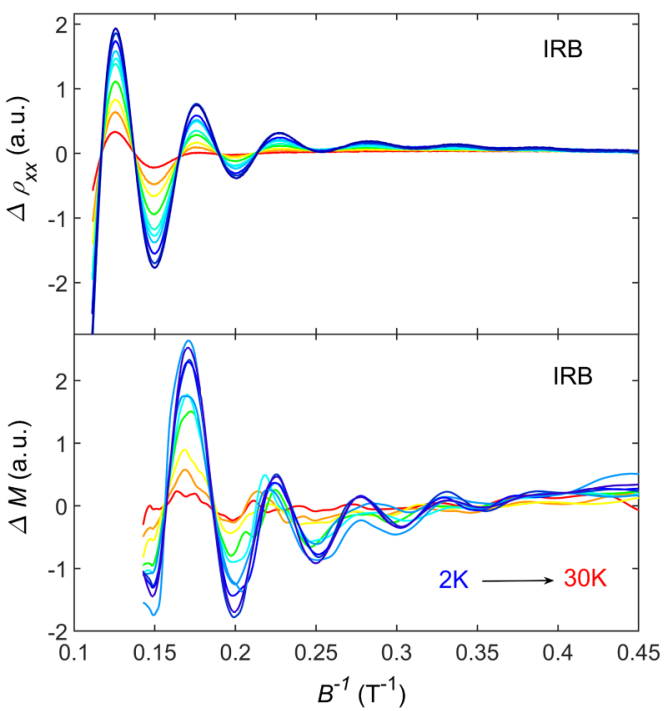

(e)

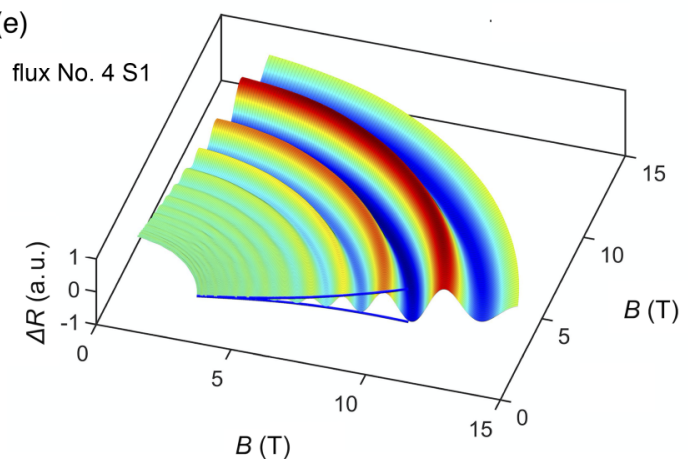

(g)

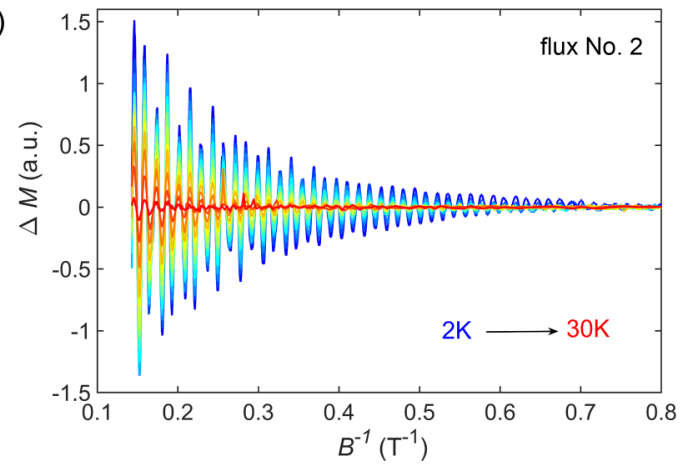

(c)

(d)

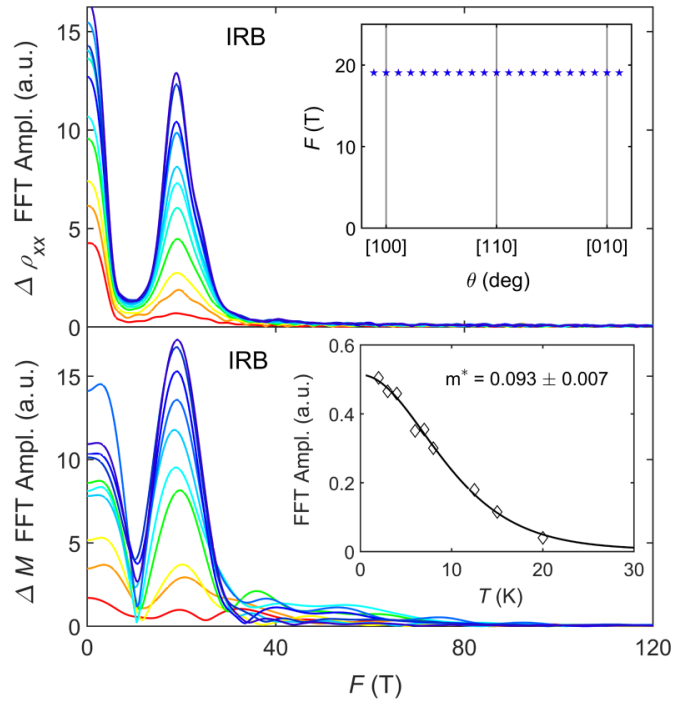

(f)

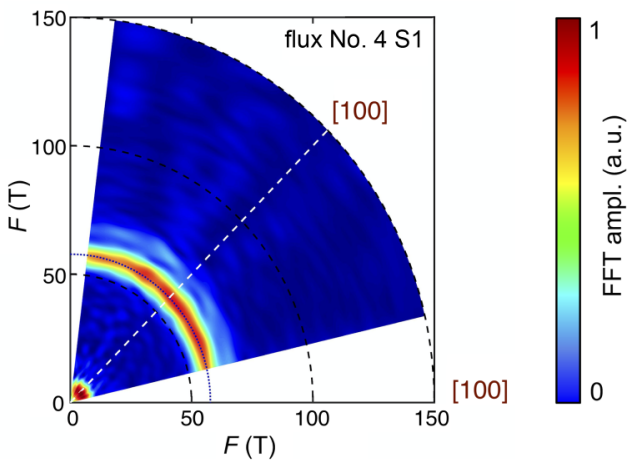

(h)

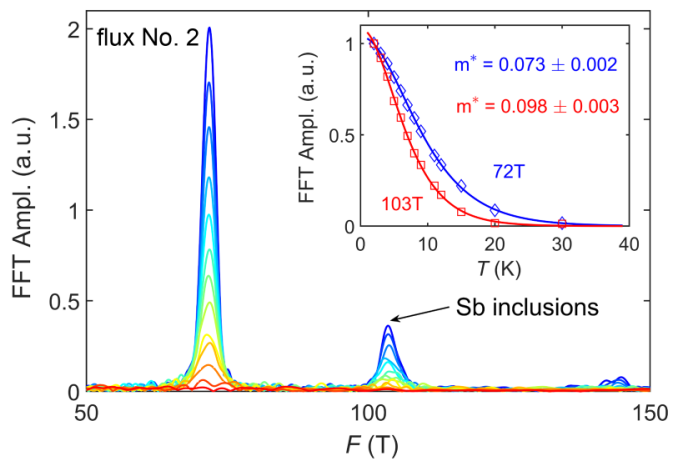

FIG. 4. Oscillatory part of the (a) resistivity and (b) magnetization of an IRB sample as a function of (c) $B^{-1}$ and the (d) corresponding FFT, respectively. Temperatures as indicated. The inset in (c) shows that the frequency is independent of the field direction. The inset in (d) depicts the temperature dependence of the oscillation amplitude from which the effective mass is extracted. (e) Angle-dependent magnetoresistance of sample flux No. $4 \mathrm{~S} 1$ at $2 \mathrm{~K}$. (f) Fourier transformation of the signal in (e). QOs and therefore FFT show an independent behavior with respect to angle, which confirms the spherical Fermi surface expected from DFT calculations. (g) dHvA oscillations from Sb inclusions in sample flux No. 2 (same data as in Fig. 2). (h) FFT over a window equivalent of $1 \mathrm{~T}$ to $7 \mathrm{~T}$ in an inverse magnetic field for the same temperatures and data in $(\mathrm{g})$ with the inset as in (d).

dures as described in the methods. The results are summarized in Table II.

First, let us examine the IRB samples. As mentioned above, QOs appear in the magnetization and resistivity as seen in Figs. 4(a) and 4(b), where the background-subtracted oscillations are shown. Their fast Fourier transforms (FFTs) are given in Figs. 4(c) and 4(d). Consistently, only one QO frequency of $19 \mathrm{~T}$ is found for this sample, but also in several samples from the same batch. The QOs stem from a spherical
Fermi surface, as expected, evidenced by a magnetic field angle-independent QO frequency [inset of Fig. 4(c)]. This oscillation frequency corresponds to a charge carrier concentration of $0.47 \times 10^{18} \mathrm{~cm}^{-3}$, which agrees with the hole concentrations of $0.55 \times 10^{18}-0.65 \times 10^{18} \mathrm{~cm}^{-3}$ from Hall measurements.

Second, we look at the flux-grown samples where two types of behavior are observed. On the one hand, in batch No. 4, a single QO frequency is observed, i.e., $57 \mathrm{~T}$ for sample $\mathrm{S} 1$ 

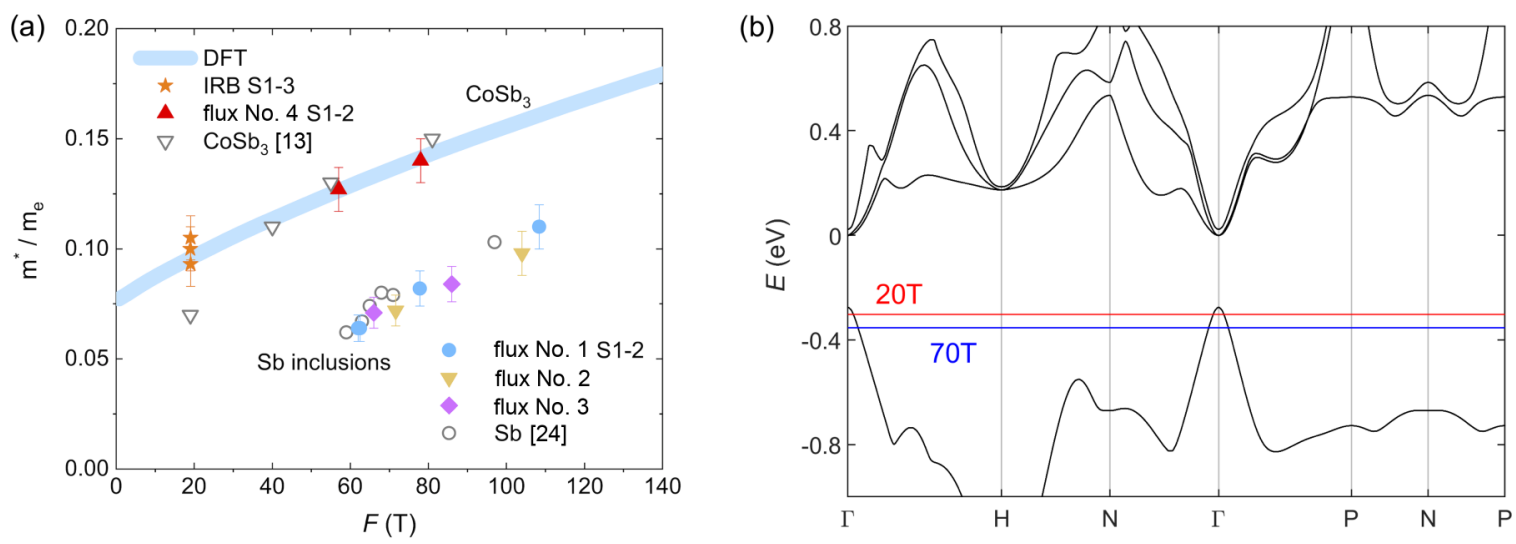

FIG. 5. (a) Effective mass $m^{*}$ in units of the free electron mass $m_{e}$ of the charge carriers as a function of the quantum oscillation frequency which depends on the Fermi energy. The light-blue line shows $m^{*}$ as determined from the DFT calculations shown in (b), with a width reflecting the variation of this line using different approximations (with/without SOC). Symbols, as indicated, give the effective masses determined for the samples of this study and from Ref. [13] (grey open triangles). Also shown is the effective mass of pure Sb [24] (grey open dots). (b) Band structure (GGA + SOC, structural parameters of sample flux No. 5 as given in Table I) with two Fermi energies corresponding to the typical observed quantum-oscillation frequencies (20 and $70 \mathrm{~T}$ ).

and $79 \mathrm{~T}$ for $\mathrm{S} 2$. The sample dependence of the QO frequency can be explained by sample-dependent doping levels, reflecting qualitatively the different carrier numbers from the Hall measurements, although the quantitative agreement is not very good here (see Table II). As in the IRB sample, the QO are not angle dependent [Figs. 4(e) and 4(f) for resistivity oscillations of sample flux No. 4 S1] and the effective mass follows the expectations of the DFT calculations for the given frequencies in Fig. 5, as discussed below.

On the other hand, dHvA oscillations of a flux-grown sample with Sb inclusions are shown in Figs. 4(g) and 4(h). Two frequencies at $72 \mathrm{~T}$ and $104 \mathrm{~T}$ can be clearly distinguished. We attribute the additional intensity in the FFT at $145 \mathrm{~T}$ to the second harmonic of the above-mentioned $72 \mathrm{~T}$ frequency. As visible in this figure and given in Table II, these samples (flux Nos. 1-3) usually show more than one frequency. The dHvA frequencies range from $60 \mathrm{~T}$ to $110 \mathrm{~T}$. The appearance of several QO frequencies can be explained in two different ways. One possibility is a sample inhomogeneity where different regions of the sample have different doping levels, i.e., hole concentrations and Fermi-surface volumes, as mentioned for samples flux No. 4 S1 and S2. Here, however, the reason lies in the previously mentioned $\mathrm{Sb}$ inclusions. Sb is a semimetal with a small and anisotropic Fermi surface and hence shows pronounced quantum oscillations with a frequency depending on the magnetic field orientation. If several single-crystalline inclusions have varying crystalline orientations, several QO frequencies should be visible. This explanation is confirmed by the analysis of the effective mass that will be discussed in the next paragraph.

Effective mass. The effective mass as a function of $\mathrm{QO}$ frequency for all samples is shown in Fig. 5(a). The samples grown by the IRB technique had a uniform effective mass of $m^{*}=0.095(10) m_{e}$ in both magnetization and resistivity quantum oscillations for various crystals (orange stars). This effective mass agrees with the prediction of the DFT calculation for this doping level (blue line) and with the behavior previously found (empty black triangles). Two samples from the batch flux No. 4 also agree with this (red triangles).
Note that this agreement with the DFT calculations points to negligible correlations in this material and hence a small renormalization of the effective mass. In the case of significant correlations, one would observe an enhanced experimental effective mass compared to the DFT calculations.

A second line of lower effective masses of flux-grown samples with QO frequencies between 60 and $110 \mathrm{~T}$ is also observed (shaded points). As indicated by the empty gray dots from Ref. [24], the behavior is in perfect correspondence with the one expected for pure $\mathrm{Sb}$ in different magnetic field orientations. Therefore, we believe that the QOs that we observe in these samples stem from $\mathrm{Sb}$ inclusions that could not be directly detected by our PXRD measurements.

\section{B. Indium doping}

Resistivity. Indium-doped samples from two growth batches with different doping level were investigated, where the batch In0.05- $\mathrm{CoSb}_{3}$ had a nominal composition $\mathrm{In}_{0.05} \mathrm{Co}_{4} \mathrm{Sb}_{12}$ and hence a lower doping than the batch In0.10-CoSb 3 with nominal composition $\mathrm{In}_{0.1} \mathrm{Co}_{4} \mathrm{Sb}_{12}$. The expansion of the lattice parameter with nominal doping shows that In is incorporated into the lattice, whereas the real doping remains unknown. The batch $\mathrm{In} 0.05-\mathrm{CoSb}_{3}$ showed an increasing resistivity upon cooling, in some samples from room temperature on and in others at lower temperatures. In none of the cases was a simple activated behavior observed.

Hall effect and charge carrier densities. The charge carrier density of the batch In0.05- $\mathrm{CoSb}_{3}$ from the Hall resistivity $\rho_{y x}$, as determined by a one-band model fit, increases towards lower temperatures by a factor of 2 . At the same time, the transverse low-temperature magnetoresistance $\Delta \rho_{x x}$ is negative, indicating the presence of an impurity band [28]. A strong influence of impurities in the materials is also indicated by an increasing rather than decreasing mobility with increasing temperature. If all indium is assumed to be incorporated homogeneously into the lattice, electronically activated, and in the $3+$ oxidation state, this is equivalent to an electron concentration of $6 \times 10^{20} \mathrm{~cm}^{-3}$ for $\mathrm{In} 0.05-\mathrm{CoSb}_{3}$. 
Clearly, the observed charge carrier densities are smaller (see Fig. 3 and Table II). This indicates a slightly inhomogeneous distribution or activation of dopants. Therefore, only one per mille to one percent of the indium is electronically incorporated into the lattice. Increasing the dopant concentration in the batch In0.10- $\mathrm{CoSb}_{3}$ raises the Fermi energy well into the electron conduction bands. Here, charge concentrations around $1 \times 10^{18}-1 \times 10^{19} \mathrm{~cm}^{-3}$ electrons were observed, which decrease by about a factor of four upon raising the temperature from $2 \mathrm{~K}$ to $300 \mathrm{~K}$, similar to what is observed in the batch $\mathrm{In} 0.05-\mathrm{CoSb}_{3}$.

From DFT calculations, three conduction bands are known. Two of these are degenerate at the $\Gamma$ point forming the direct gap, while the third is gapped [25]. Since thermal excitations between the bands, and from a possible impurity band, into the higher conductance bands are possible with increasing temperature, the change in mobilities and charge carrier density is attributed to multiband effects.

Quantum oscillations. In three samples of the batch In0.10-CoSb 3 , Shubnikov-de Haas and dHvA oscillations with frequencies around $(25 \pm 3) \mathrm{T}$ and $(40 \pm 3) \mathrm{T}$ for $B \|$ [100] could be observed, whereas the batch $\mathrm{In} 0.05-\mathrm{CoSb}_{3}$ showed none. The calculation predicts that three Fermi surfaces should be present for the electron-doped material. Of the two detectable frequencies, the lower one was too faint to be analyzed further. For the larger observed frequency, only two or three maxima of the oscillations appeared at highest fields, rendering a Fourier transformation not ideal for their analysis. Therefore, the more cumbersome approach of fitting the resistivity with the Lifshitz-Kosevich equation has been used (see Ref. [29]). This revealed the angular dependence given in Fig. 6(a). It was fitted by a cubic harmonic expansion of the Fermi surface; the result is shown in Fig. 6(b). It matches the second-largest Fermi surface from DFT calculations for the undoped crystal, when the Fermi energy is moved into the electron-doped region with $E_{F}=0.16 \mathrm{eV}$, as given in Fig. 6(c). This again confirms the reliability of the DFT calculations for this compound.

\section{SUMMARY}

We succeeded in growing $\mathrm{CoSb}_{3}$ single crystals out of an $\mathrm{Sb}$ flux and by an inclined rotary Bridgman method. As-grown samples of this small-gap semiconductor appear metallic with small amounts of hole carriers putting the Fermi energy to the hole band. The ensemble of our measurements shows that samples grown by the IRB technique are homogeneous. The Fermi level is near the band edge, producing a small spherical Fermi surface observed by a unique QO frequency. The Fermi level in as-grown samples by flux is lower, whereas indium doping pushes the Fermi level into the conduction band. Experimental Fermi-surface properties are consistent with DFT calculations and previous experimental investigations. Some flux-grown samples also show quantum oscillations from $\mathrm{Sb}$ inclusions, which could be distinguished via the lower effec-

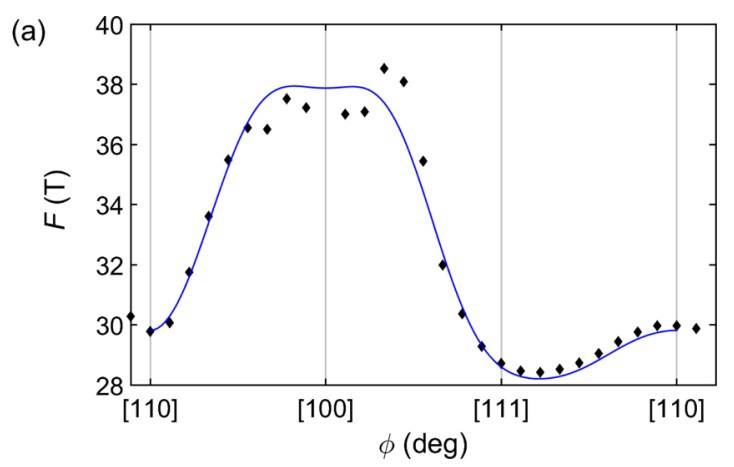

(b)

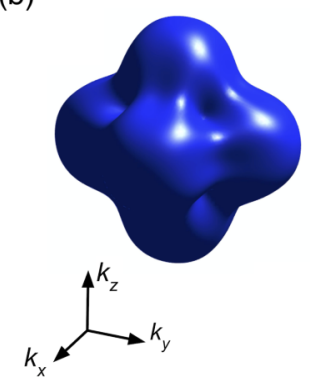

(c)

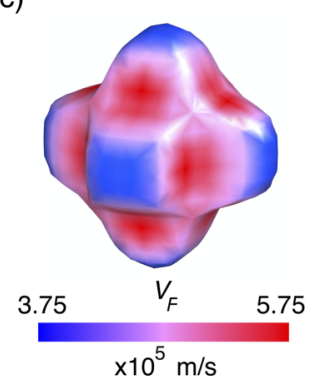

FIG. 6. Results of the quantum-oscillation study on the indiumdoped $\mathrm{CoSb}_{3}$ sample, In0.10-CoSb . (a) The angular dependence of the higher of two observed quantum-oscillation frequencies. (b) The reconstructed Fermi surface via a cubic harmonic expansion, fitted to the angular dependence of the $\mathrm{SdH}$ frequencies [blue line in (a)]. (c) The DFT Fermi surface for $E_{\mathrm{f}}=0.16 \mathrm{eV}$ of the second of the three electron bands. Colors indicate the Fermi velocity.

tive mass. Therefore, we can confirm the reliability of the DFT calculations experimentally at ambient conditions in $\mathrm{CoSb}_{3}$ for Fermi levels close to the gap. These samples and results are identified as a good starting point for future experiments under strain, where the transition to a topological insulator phase is expected.

\section{ACKNOWLEDGMENTS}

We would like to thank M. Brando and A. Steppke for help with the transport measurements in their cryostat, and H. Rave and C. Klausnitzer for technical support. U. Nitzsche (IFW Dresden) is acknowledged for technical support. M.N. thanks J. Klotz for stimulating discussions. The authors acknowledge support from the Max Planck Society (Max-Planck-Gesellschaft) for the Max Planck research group "Physics of Unconventional Metals and Superconductors." We acknowledge funding via Project E3 of the TRR80 "From electronic correlations to functionality" (Deutsche Forschungsgemeinschaft Project No. 107745057). M.P. acknowledges Ph.D. funding from the Peruvian Council of Science and Technology (CONCYTEC) under Grant No. 2212014-FONDECYT.

[1] E. D. Bauer, N. A. Frederick, P. -C. Ho, V. S. Zapf, and M. B. Maple, Phys. Rev. B 65, 100506(R) (2002).
[2] B. C. Sales, D. Mandrus, and R. K. Williams, Science 272, 1325 (1996). 
[3] J. C. Smith, S. Banerjee, V. Pardo, and W. E. Pickett, Phys. Rev. Lett. 106, 056401 (2011).

[4] B.-J. Yang and N. Nagaosa, Nat. Commun. 5, 4898 (2014).

[5] D. E. Sheehy and J. Schmalian, Phys. Rev. Lett. 99, 226803 (2007).

[6] P. Sun, B. Wei, J. Zhang, J. M. Tomczak, A. M. Strydom, M. Søndergaard, B. B. Iversen, and F. Steglich, Nat. Commun. 6, 7475 (2015).

[7] H. Rakoto, E. Arushanov, M. Respaud, J. M. Broto, J. Leotin, C. Kloc, E. Bucher, and S. Askenazy, Phys. B: Condens. Matter 246-247, 528 (1998).

[8] T. Caillat, A. Borshchevsky, and J. Fleurial, J. Appl. Phys. 80, 4442 (1996).

[9] D. Mandrus, A. Migliori, T. W. Darling, M. F. Hundley, E. J. Peterson, and J. D. Thompson, Phys. Rev. B 52, 4926 (1995).

[10] J. W. Sharp, E. C. Jones, R. K. Williams, P. M. Martin, and B. C. Sales, J. Appl. Phys. 78, 1013 (1995).

[11] E. Arushanov, K. Fess, W. Kaefer, C. Kloc, and E. Bucher, Phys. Rev. B 56, 1911 (1997).

[12] D. T. Morelli, T. Caillat, J.-P. Fleurial, A. Borshchevsky, J. Vandersande, B. Chen, and C. Uher, Phys. Rev. B 51, 9622 (1995).

[13] E. Arushanov, M. Respaud, H. Rakoto, J. M. Broto, and T. Caillat, Phys. Rev. B 61, 4672 (2000).

[14] D. J. Singh and W. E. Pickett, Phys. Rev. B 50, 11235 (1994).

[15] M. Pillaca, O. Harder, W. Miller, and P. Gille, J. Cryst. Growth 475, 346 (2017).
[16] L. Akselrud, P. Zavalii, Y. Grin, V. Pecharski, B. Baumgartner, and E. Wölfel, MSF 133-136, 335 (1993).

[17] K. Koepernik and H. Eschrig, Phys. Rev. B 59, 1743 (1999).

[18] I. Opahle, K. Koepernik, and H. Eschrig, Phys. Rev. B 60, 14035 (1999).

[19] J. P. Perdew, K. Burke, and M. Ernzerhof, Phys. Rev. Lett. 77, 3865 (1996).

[20] J. P. Perdew and Y. Wang, Phys. Rev. B 45, 13244 (1992).

[21] H. Eschrig, M. Richter, and I. Opahle, J. Theor. Comput. Chem. 14, 723 (2004).

[22] J.-F. Mercure, The de Haas-van Alphen effect near a quantum critical end point in Sr3Ru2O7, Ph.D. thesis, University of St. Andrews, 2008.

[23] F. Arnold, M. Naumann, S. Khim, H. Rosner, V. Sunko, F. Mazzola, P. D. C. King, A. P. Mackenzie, and E. Hassinger, Phys. Rev. B 96, 075163 (2017).

[24] Y. Ishizawa, J. Phys. Soc. Jpn. 25, 150 (1968).

[25] See Supplemental Material at http://link.aps.org/supplemental/ 10.1103/PhysRevB.103.085133 for the band structure calculated using different approximations.

[26] B. L. Altshuler and A. G. Aronov, in Modern Problems in Condensed Matter Sciences, Electron-Electron Interactions in Disordered Systems, Vol. 10, edited by A. L. Efros and M. Pollak (Elsevier, Amsterdam, 1985), pp. 1-153.

[27] D. Long and J. Myers, Phys. Rev. 115, 1107 (1959).

[28] J. F. Woods and C. Y. Chen, Phys. Rev. 135, A1462 (1964).

[29] I. Lifshitz and A. Kosevich, J. Exper. Theoret. Phys. USSR 29, 730 (1956) [JETP 2, 636 (1956)]. 